\title{
Mannheim Carotid Intima-Media Thickness and Plaque Consensus (2004-2006-2011)
}

An Update on Behalf of the Advisory Board of the 3rd, 4th and 5th Watching the Risk Symposia, at the 13th, 15th and 20th European Stroke Conferences, Mannheim, Germany, 2004, Brussels, Belgium, 2006, and Hamburg, Germany, 2011

\author{
P.-J. Touboul M.G. Hennerici S. Meairs H. Adams P. Amarenco N. Bornstein
}

L. Csiba M. Desvarieux S. Ebrahim R. Hernandez Hernandez M. Jaff

S. Kownator T. Naqvi P. Prati T.Rundek M.Sitzer U.Schminke J.-C. Tardif

A. Taylor E. Vicaut K.S. Woo

\section{Key Words}

Intima-media thickness $\cdot$ Carotid plaque $\cdot$ Vascular ultrasound · Randomized clinical trials • Carotid disease • Reference values

\begin{abstract}
Intima-media thickness (IMT) provides a surrogate end point of cardiovascular outcomes in clinical trials evaluating the efficacy of cardiovascular risk factor modification. Carotid artery plaque further adds to the cardiovascular risk assessment. It is defined as a focal structure that encroaches into the arterial lumen of at least $0.5 \mathrm{~mm}$ or $50 \%$ of the surrounding IMT value or demonstrates a thickness $>1.5 \mathrm{~mm}$ as measured from the media-adventitia interface to the intima-lumen interface. The scientific basis for use of IMT in clinical trials and practice includes ultrasound physics, technical and disease-related principles as well as best practice on the performance, interpretation and documentation of study results. Comparison of IMT results obtained from epidemiological and interventional studies around the world relies on
\end{abstract}

harmonization on approaches to carotid image acquisition and analysis. This updated consensus document delineates further criteria to distinguish early atherosclerotic plaque formation from thickening of IMT. Standardized methods will foster homogenous data collection and analysis, improve the power of randomized clinical trials incorporating IMT and plaque measurements and facilitate the merging of large databases for meta-analyses. IMT results are applied to individual patients as an integrated assessment of cardiovascular risk factors. However, this document recommends against serial monitoring in individual patients.

Copyright $\odot 2012$ S. Karger AG, Basel

\section{Introduction}

Progressive, subclinical arterial wall alterations precede cardiovascular clinical events, which then reflect advanced atherosclerotic disease. The first morphological abnormalities of arterial walls can be visualized by B-mode ultrasonography. This high-resolution, noninva-

\section{KARGER}

Fax +41613061234 E-Mail karger@karger.ch www.karger.com

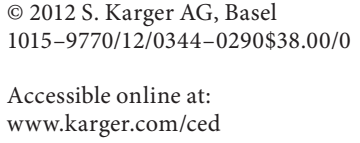


sive technique provides one of the best methods for the detection of early stages of atherosclerotic disease, based upon its simple nature, wide availability, and demonstrated capacity to depict arterial wall structure with better resolution than any other similar technique (e.g. magnetic resonance imaging or radiographic techniques). Accordingly, many studies have successfully applied intimamedia thickness (IMT) as a technique to monitor arterial wall alterations based upon its association with cardiovascular risk factors and the incident cardiovascular disease [1-6]. However, these measurements are not strictly associated with individual risks of stroke, myocardial infarction and peripheral artery disease.

Diverse approaches for measuring IMT exist, creating confusion in the literature. In addition, unified criteria are needed for distinguishing atherosclerosis as seen in early plaque formation from thickening of the intimamedia complex on the recognition that IMT reflects not only early atherosclerosis, but also nonatherosclerotic compensatory remodeling with largely medial hypertrophy as a result of smooth muscle cell hyperplasia and fibrocellular hypertrophy. This differentiation is important because epidemiological studies have shown that wall thickening as depicted by ultrasonographic measurements of IMT is different from atherosclerotic plaque regarding localization, natural history, risk factors and predictive value for vascular events.

As IMT is increasingly used in clinical trials to serve as a surrogate end point for best medical treatment, the success of interventions that lower risk factors for atherosclerosis can be documented. Further advancement for the field of IMT requires standardized methods, enabling homogenous data collection and analysis. This may help to improve the power of such studies and to facilitate the merging of large databases for meta-analyses.

This update of the last Mannheim IMT Consensus Document [7] addresses the important issue of standardization of carotid IMT measurements and seeks to clarify problems related to the classification of early atherosclerotic lesions.

\section{Distinction between IMT and Plaque}

In the absence of atherosclerotic plaques, B-mode ultrasound displays the vascular wall as a regular pattern that correlates with anatomical layers. The area of tissue starting at the luminal edge of the artery and ending at the boundary between the media and the adventitia represents the intima-media portion of this pattern.

Mannheim Carotid Intima-Media

Thickness and Plaque Consensus
This interface is well depicted by ultrasound. With increasing age, this pattern thickens in a uniform way within straight arterial segments. All known major vascular risk factors contribute to increased IMT thickening and its progression. As a mirror of these processes, IMT provides a tool to investigate normal aging and preclinical atherosclerosis. Ultrasound imaging further identifies later stages of atherosclerosis (plaque, stenosis, occlusion) which may also be identified either in the absence of or coincident with increased IMT. However, intermediate stages between increasing IMT and atherosclerotic plaque formation cannot clearly be differentiated neither by ultrasound nor by histological examination.

Such conditions are common at the bifurcation and the origin of the internal carotid artery, but occur only occasionally in the common carotid artery (CCA) where most studies are performed. Epidemiological and intervention studies have shown that although both share common risk factors of atherosclerosis, its natural history, patterns of risk factors and the prediction of cardiac and cerebral events are different for carotid IMT and carotid plaque [8-10].

\section{Definitions for Ultrasound Characterization of IMT and Atherosclerotic Plaque}

Ultrasound techniques identify:

(1) IMT is a double-line pattern visualized by echography on both walls of the CCA in a longitudinal image. Two parallel lines, which consist of the leading edges of two anatomical boundaries, form it: the lumen-intima and media-adventitia interfaces. This definition complies with the IMT correlation studies comparing this ultrasound pattern to carotid anatomy specimens [11] (fig. 1).

(2) Plaques are focal structures encroaching into the arterial lumen of at least $0.5 \mathrm{~mm}$ or $50 \%$ of the surrounding IMT value, or demonstrates a thickness $>1.5 \mathrm{~mm}$ as measured from the intima-lumen interface to the mediaadventitia interface.

These definitions permit classification of the vast majority of carotid lesions observed with ultrasound.

\section{Physical and Structural Basics}

Carotid examination includes visualization of common, internal and external carotid arteries. Continuous technical progress among ultrasound devices now pro- 


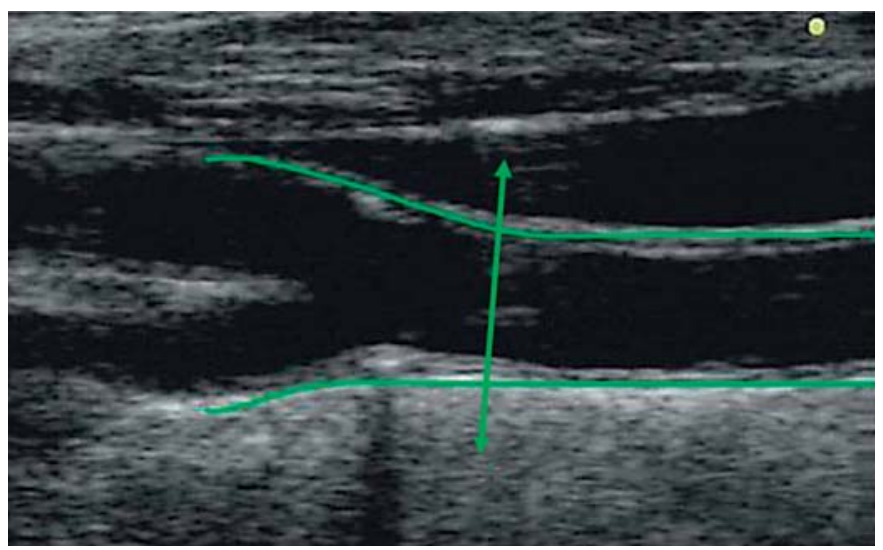

Fig. 1. Longitudinal view of CCA and carotid bifurcation and origin of internal and external carotid arteries. The double arrow line corresponds to the end of the CCA, where near and far walls start diverging.

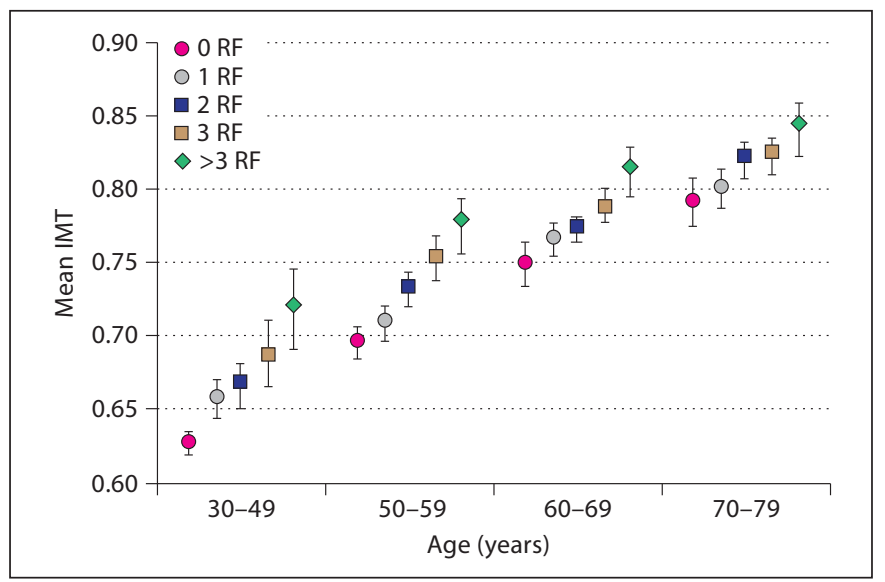

Fig. 2. Age-related quartiles of risk factors (RF). No patients between 30 and 39 years have $>3$ risk factors.

vides higher spatial and density resolutions. Ongoing technical progress includes the emergence of three-dimensional imaging.

\section{Ultrasound Principles of Physics and Technology}

- The virtual biconcave lens of an ultrasound beam with its thicker nature on the near and distal part of the field leads to the optimal resolution in the mid part of this field.

- Establishing the perpendicular relationship between the ultrasound beam and the visualized structures provides optimal reflection of the incident ultrasound beam.
- The energy of the ultrasound beam decreases with the depth of insonation making the far field darker.

- The probe frequency has a direct relationship to resolution, and an inverse relationship to depth of ultrasound penetration.

- Linear transducers activate piezo-electric crystals simultaneously resulting in a synchronized propagation of the ultrasound wave. Sector scanners are typically activating crystals sequentially in predefined time intervals, causing asymmetric shifts and reception of skewed wavefronts. Linear ultrasound transducers provide best image quality for superficial arteries, while electronic sector scanners are better used for deep structures with less accessibility.

- B-mode signals which are displayed on the screen derive from the received raw radiofrequency signals of the probe.

\section{Anatomic Structures and Physiological Principles}

- Common and internal carotid arteries are homogeneous in structure and hemodynamics.

- Insonation is limited at the origin by the brachioarterial cephalic trunk on the right side and the aortic arch on the left side, the CCA ends at the bifurcation that is represented anatomically by the point of divergence of the CCA walls (fig. 1).

- The bifurcation is rather heterogeneous between individuals. At this site, remodeling varies according to the angles, curvatures and diameters of carotid arteries that influence shear stress constraints and modify wall thickness independently of any arterial disease or risk factor $[12,13]$.

- Since atherosclerosis usually starts in the carotid bulb, delineation of the different segments is required.

\section{Standards for Image Acquisition}

\section{Intima Media Thickness}

Carotid arterial wall assessment may include the common, internal or bulb segments of the carotid artery. Whereas nearly all patients have their CCA imaged, successful imaging of the internal carotid artery and of the carotid bulb depends both upon the anatomical topography of the patient and on sonographer's expertise.

Settings of the Ultrasound Device

(1) Standard equipment includes a high-resolution Bmode system operating in the black and white mode, 
preferentially with linear ultrasound transducers at frequencies $>7 \mathrm{MHz}$.

(2) Appropriate depth of focus (e.g. 30-40 mm), and optimal frame rate $25 \mathrm{~Hz}(>15 \mathrm{~Hz})$ provide optimal image quality and facilitate edge detection.

(3) Log gain compensation of approximately $60 \mathrm{db}$.

(4) Gain settings adjusted to obtain a symmetrical brightness on the near and far wall, or in the mid part of the field to eliminate intraluminal artifacts.

Examination Procedure

(1) Arterial wall segments should be assessed in a longitudinal view, strictly perpendicular to the ultrasound beam.

(2) Both walls should be clearly visualized in order to achieve diameter measurements. The optimal diameter should be obtained during diastole by automatic cineloop detection or by looking for the minimal diameter during the cardiac cycle (fig. 4).

(3) Measurements obtained from radiofrequency signals are equivalent to those obtained with conventional video signals $[14,15]$.

(4) Lateral probe position is recommended as it offers the best resolution in the midfield.

(5) Acquisition of multi-insonation angles increases procedure time consumption without benefit for measurements; however, in clinical trials they result in better reproducibility and facilitate statistical analysis [16].

(6) Horizontal arterial image display optimizes the interfaces between blood and vessel wall structures.

(7) Imaging of the carotid bifurcation provides a landmark essential in serial imaging.

(8) Longitudinal and cross-sectional views are required to visualize focal atherosclerosis.

Plaque acquisition should be done along the carotid tree, in longitudinal and cross-sectional views. Color flow Doppler imaging helps to identify low echogenic boundaries.

\section{Measurement Location}

Measurement of IMT should occur within a region free of plaque with a clearly identified double-line pattern. Such measurement will have increased accuracy, reproducibility [17], and ease of measurement using computer-based techniques. Such measurements will match a majority of reference values within studies performed throughout the world. The majority of reference values provide data from different countries [18-20].

Mannheim Carotid Intima-Media

Thickness and Plaque Consensus

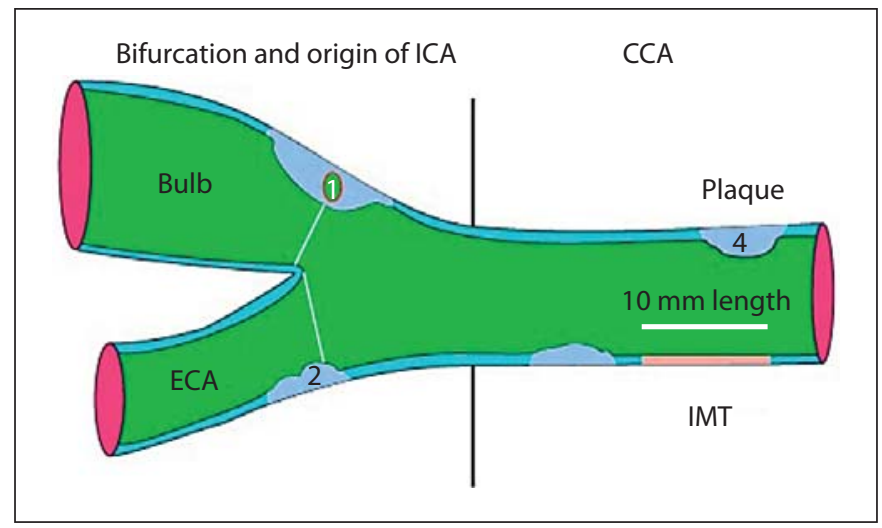

Fig. 3. Drawn representation of carotid tree, with plaque and IMT measurement according to Mannheim consensus: (1) thickness $>1.5 \mathrm{~mm}$; (2) lumen encroaching $>0.5 \mathrm{~mm} ;(3,4)>50 \%$ of the surrounding IMT value.

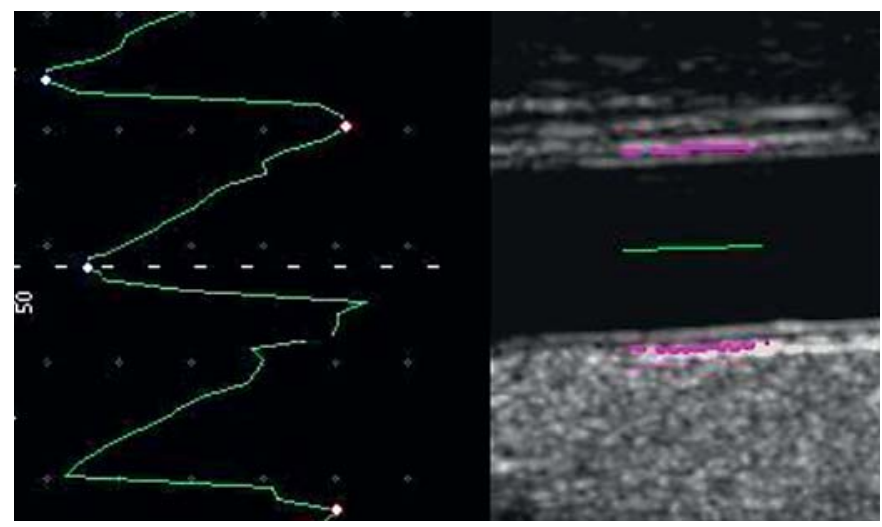

Fig. 4. Automatic selection of the end diastole frame from a CCA video acquisition for an IMT measure.

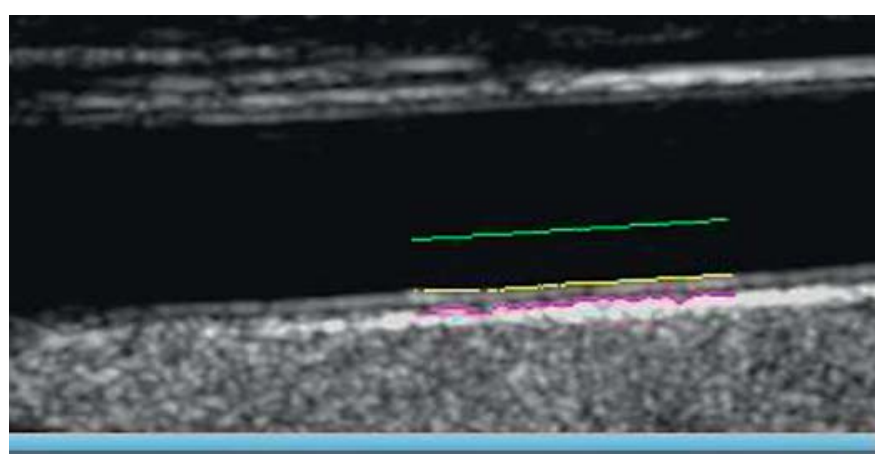

QI 94 Maximal: $0.788 \mathrm{~mm}$ Mean: $0.625 \mathrm{~mm}$

Fig. 5. IMT measurement in diastole on the selected frame. QI = Quality Index indicates that 94\% of 150 measures could be performed. Maximal is the highest value obtained from all the elementary measures. Mean is the average of all the measurements that could be performed.

Cerebrovasc Dis 2012;34:290-296 
This pattern is the only one that has been validated with anatomy [11].

(1) IMT should be measured preferably on the far wall of the CCA at least $5 \mathrm{~mm}$ below its end (fig. 3-5) which avoids interindividual variability induced by physiological remodeling and less gain dependence will exist. Values from the near wall depend in part on gain settings and are less reliable. If taken on the near wall, its value should be recorded separately from IMT of the far wall.

(2) IMT can be measured at the carotid bifurcation or ICA bulb in a region free of plaque, on a shorter length, taking caution of the large interindividual variability induced by remodeling and anatomic variations. These values must be recorded separately [21]. In study designs that include wall thickness, values obtained from different sites of the carotid arteries should be documented separately.

(3) Along $10 \mathrm{~mm}$ length of a straight arterial segment, a high-quality image acquisition is required for reproducible serial measurements. In case of vessel tortuosity, IMT measurements are only possible at a shorter vessel segment, especially in the carotid bifurcation or the ICA bulb.

(4) For plaque: Maximal thickness requires demonstration from 2 different angles of insonation, in longitudinal and cross-sectional views.

\section{Methodology Standards}

\section{IMT}

(1) Edge detection systems provide accurate measurements of IMT [22]. Although manual measurements may lead to valid measurements, they require rigorous quality control and quality assurance. Manual and semimanual reading methods require more time than automated systems. Automated systems can provide the mean maximal value of 150 measurements performed on a $10-\mathrm{mm}$ segment of CCA instantaneously.

Manual measurements are more observer dependent than semi-automatic systems.

(2) CCA inter-adventitial and intraluminal diameters must also be measured as IMT is significantly correlated with arterial diameter [23].

(3) IMT measurements options include the mean, maximum, composite measures from both sides and different arterial sites. Mean IMT values averaged across the entire distance are less susceptible to outliers, whereas the maximal IMT may reflect more advanced stages with focal thickening or plaque formation. The maximal value may be misleading due to the effect of measurement and sampling errors.
IMT values from the left and right side can be averaged although there is a significant difference between the left and right CCA IMT, with higher values on the left side [24]. Composite scores including both plaque and IMT should be avoided.

(4) Each vascular laboratory must perform quality control of their equipment periodically by (phantom scans) and reliability studies of scans and measurements for ultra-sonographers and readers. Reporting requirements include the intra-class correlation coefficients to be evaluated for intra- and interobserver variability, both for IMT and plaque measurements.

\section{Plaque}

(1) Plaque location, thickness, area and number, scanned in longitudinal and cross-sections must be recorded. The incremental value of recording texture (density, echogenicity, shadow) remains uncertain pending more research.

\section{Guidelines and Use in Clinical Practice}

Guidelines that support the use of IMT for the assessment of cardiovascular risk include: ACC/AHA Guideline on the initial detection of cardiovascular risk: Recommends assessment as a class IIA ('is reasonable to perform') in individual at intermediate coronary heart disease risk [25].

The National Cholesterol Education program Adult Treatment Panel III identified IMT as a method to detect subclinical atherosclerosis and guide selection and intensification of lipid management [26].

The ESH/ESC European practice guidelines in Spain include IMT to detect target organ damage induced by hypertension [27].

The American Society of Echocardiography [28] and the Society of Atherosclerosis Imaging and Prevention [29] published appropriate use criteria.

CIMT and plaque presence are recommended for the initial detection of CHD risk in asymptomatic patients:

- at intermediate risk

- in the setting of 2 or more NCEP risk factors

- with metabolic syndrome

- in the setting of a family history of premature CHD

- with a known CAC score of zero and FRS 11-20\%. 


\section{Reference Values and Societies Guidelines}

The differences observed in IMT values in a healthy population from different countries are related to many factors, including methodological variability and differences in cardiovascular risk and factor profiles [30-32].

Since 2000, several guidelines suggest that carotid artery B-mode ultrasound imaging is safe, noninvasive and relatively inexpensive allowing to assess subclinical atherosclerosis [33-35] in asymptomatic persons $>45$ years old and could add incremental information to traditional risk factor assessment [36-39]. Reference values require matching to specific imaging protocols utilized in large sample size population as much as possible.

Risk calculators may integrate IMT measurement with risk factor measurements. The ARIC study has published such a calculator incorporating IMT and plaque assessment to determine an adjusted Framingham risk. CCAIMT 'normal values' in the absence of plaque should help to characterize populations at intermediate risk.

\section{Use of IMT in Research}

Epidemiological and interventional trials evaluating cardiovascular diseases should include IMT and plaque to better characterize the study population.

Ancillary studies in research should be sufficiently powered to evaluate the predictive value of IMT and plaque for incident clinical events.

In clinical trials, several additional requirements are recommended to assure data harmonization. They can be performed through an expert IMT/Plaque panel as usually represented in the Steering Committees [40].

Clinical trials using IMT and plaque as intermediate outcomes need to address the following:

- Study design.

- Study quality control.

- Full image monitoring.

- Statistical plan analysis.

- Data monitoring (from CRO and Core Lab).

- Result analysis and definition:

(1) Inclusion criteria for the participating ultrasound centers.

(2) Equipment installation if necessary.

(3) Sonographers training and performance control.

(4) Certification of the centers (equipment and sonographers).
(5) Quality assurance and quality control.

(6) Central reading facilities.

- Evaluation of data variability through intra-class correlation coefficient.

- Choice of end points depends on:

(1) Primary or secondary prevention status.

(2) Epidemiological or interventional studies.

IMT and plaque measurements including maximal or mean IMT, plaque thickness, area and volume, and plaque score may all be useful as imaging outcomes.

All these procedures and choices can reduce measurement variability, which is a key parameter for a highquality study, statistical power and sample size determination.

\section{Conclusions}

Carotid IMT and plaques are different phenotypes indicating increased vascular risk. Plaque presence demonstrates a higher risk and therefore overrides IMT predictive values. However, IMT without plaque remains a significant marker of an increased risk of vascular events and significantly predicts plaque occurrence. The continuity of vascular wall changes is best monitored in CCA IMT studies, different from discontinuous focal lesions (plaque) which are characteristic of atherosclerotic disease. Therefore, the distinction between IMT and plaque must be clearly specified in the scanning protocols.

References Magyar MT, Szikszai Z, Balla J, et al: Early-
onset carotid atherosclerosis associated with increased intima media thickness and elevated serum levels of inflammatory markers. Stroke 2003:34:58-63.

$\checkmark 2$ Luedemann J, Schminke U, Berger K, et al: Association between behavior-dependent cardiovascular risk factors and asymptomatic carotid atherosclerosis in a general population. Stroke 2002;33:2929-2935.

3 Lorenz MW, von Kegler S, Steinmetz H, et al: Carotid intima-media thickening indicates a higher vascular risk across a wide age range: prospective data from the Carotid Atherosclerosis Progression Study (CAPS). Stroke 2006:37:87-92.

-4 Van der Meer IM, Iglesias del Sol A, Hak AE, et al: Risk factors for progression of atherosclerosis measured at multiple sites in the arterial tree: The Rotterdam Study. Stroke 2003;34:2374-2379. 
-5 Touboul PJ, Elbaz A, Koller C, Lucas C, Adrai V Chedru F, Amarenco P: Common carotid artery intima-media thickness and brain infarction: The 'Etude du Profil Génétique de l'Infarctus Cérébral' (GENIC) casecontrol study. The GENIC Investigators. Circulation 2000;102:313-318.

-6 Lorenz MW, Markus S, Bots M-L, et al: A systematic review and meta-analysis: prediction of clinical cardiovascular events with carotid intima-media thickness. Circulation 2007;115:459-467.

-7 Touboul PJ, Hennerici MG, Meairs S, Adams $\mathrm{H}$, Amarenco P: Mannheim Carotid IntimaMedia Thickness Consensus (2004-2006): An Update on Behalf of the Advisory Board of the 3rd and 4th Watching the Risk Symposium 13th and 15th European Stroke Conferences, Mannheim, Germany, 2004, and Brussels, Belgium, 2006. Cerebrovasc Dis 2004;18:346-349.

-8 Ebrahim S, Papacosta O, Whincup P, et al: Carotid plaque, intima media thickness, cardiovascular risk factors, and prevalent cardiovascular disease in men and women: the British Regional Heart Study. Stroke 1999; 30:841-850.

-9 Zureik M, Ducimetière P, Touboul PJ, et al: Common Carotid Intima-Media Thickness Predicts Occurrence of Carotid Atherosclerotic Plaques: Longitudinal Results from the Aging Vascular Study (EVA) study. Arterioscler Thromb Vasc Biol 2000;20:1622-1629.

10 von Sarnowski B, Lüdemann J, Völzke H, et al: Common carotid artery intima-media thickness and Framingham risk score predict incident carotid atherosclerosis plaque formation: longitudinal results from the SHIP study (Study of Health In Pomerania). Stroke 2010;41:2375-2377.

11 Pignoli P, Tremoli E, Poli A, et al: Intimal plus medial thickness of the arterial wall: a direct measurement with ultrasound imaging. Circulation 1986;74:1399-1406.

12 Glagov S, Weisenberg E, Zarins CK, et al: Compensatory enlargement of human atherosclerotic coronary arteries. $\mathrm{N} \mathrm{Engl} \mathrm{J} \mathrm{Med}$ 1987;316:1371-1375.

13 Steinke W, Els T, Hennerici M: Compensatory carotid artery dilatation in early atherosclerosis. Circulation 1994;89:2578-2581.

14 Dogan S, Plantinga Y, Dijk JM, et al, SMART Study Group: B-mode versus automated radio-frequency carotid intima-media thickness measurements J Am Soc Echocardiogr 2009;22:1137-1144.

15 Urbina E, Sathanur R: Impact of multiple coronary risk factors on the intima-media thickness of different segments of carotid artery in healthy young adults (The Bogalusa Heart Study). Am J Cardiol 2002;90:953958.

16 Dogan S, Duivenvoorden R, Grobbee D, et al, on Behalf of the Radiance 1 and Radiance 2 Study Groups: Ultrasound protocols to measure carotid intima-media thickness in trials; comparison of reproducibility, rate of progression, and effect of intervention in subjects with familial hypercholesterolemia and subjects with mixed dyslipidemia. Ann Med 2010;42:447-464.

17 Gonzalez J, Wood JC, Dorey FJ, et al: Reproducibility of carotid intima-media thickness measurements in young adults. Radiology 2008;247:2.

18 Tosetto A, Prati P, Baracchini C, et al: Ageadjusted reference limits for carotid intimamedia thickness as better indicator of vascular risk: population-based estimates from the VITA project. J Thromb Haemost 2005; 3:1224-1230.

19 Howard G, Sharrett AR, Heiss G, et al: Carotid artery intimal-medial thickness distribution in general populations as evaluated by B-mode ultrasound. ARIC Investigators. Stroke 1993;24:1297-1304.

20 Touboul PJ, Labreuche J, Vicaut E, et al: Country-based reference values and impact of cardiovascular risk factors on carotid intima-media thickness in a French population: The 'Paroi Artérielle et Risque CardioVasculaire' (PARC) Study. Cerebrovasc Dis 2009;27:361-367.

21 Kanters S, Algra A, van Leuween MS, et al: Reproducibility of in vivo carotid intimamedia thickness measurements: a review. Stroke 1997;28:665-671.

22 Touboul PJ, Prati P, Scarabin PY, et al: Use of monitoring software to improve the measurement of carotid wall thickness by Bmode imaging. J Hypertens Suppl 1992; 10:S37-S41.

23 Wikstrand J: Methodological considerations of ultrasound measurement of carotid artery intima-media thickness and lumen diameter. Clin Physiol Funct Imaging 2007;27:341-345.

24 Foerch C, Buehler A, von Kegler S, et al: Intima-media thickness side differences are limited to the common carotid artery. Hypertension 2003;42:e17.

$\checkmark 25$ Greenland P, Alpert JS, Beller GA: ACCF/ AHA guideline for assessment of cardiovascular risk in asymptomatic adults: a report of the American College of Cardiology Foundation/American Heart Association Task Force on Practice Guidelines. J Am Coll Cardiol 2010;56:e50-e103.

26 National Cholesterol Education Education Program (NCEP) Expert Panel (ATP III): Third report of the National Cholesterol Education Program (NCEP) Expert Panel on Detection, Evaluation, and Treatment of High Blood Cholesterol in Adults (Adult Treatment Panel III) final report. Circulation 2002;106:143-421.

27 de la Sierra A, Zamorano JL, Ruilope LM: Application of hypertension guidelines in clinical practice: implementation of the 2007 ESH/ESC European Practice Guidelines in Spain. J Hypertens Suppl 2009;27:S27-S32.

28 Stein JH, Korcarz CE, Hurst RT, et al: Use of carotid ultrasound to identify subclinical vascular disease and evaluate cardiovascular disease risk: a consensus statement from the American Society of Echocardiography Carotid Intima-Media Thickness Task Force.
Endorsed by the Society for Vascular Medicine. American Society of Echocardiography Carotid Intima-Media Thickness Task Force. J Am Soc Echocardiogr 2008;21:93111; quiz 189-190.

29 Society of Atherosclerosis Imaging and Prevention, Developed in collaboration with the International Atherosclerosis Society: Appropriate use criteria for carotid intima thickness testing. Atherosclerosis 2011;214:43-46.

30 Bis JC, Kavousi M, Francheschini N, et al: CARDIoGRAM Consortium. Meta-analysis of genome-wide association studies from the CHARGE consortium identifies common variants associated with carotid intima media thickness and plaque. Nat Genet 2011;43: 940-947.

-31 Rundek T, Elkind MS, Pittman J, et al: Carotid intima-media thickness is associated with allelic variants of stromelysin-1, interleukin-6, and hepatic lipase genes: the Northern Manhattan Prospective Cohort Study. Stroke 2002;33:1420-1423.

32 Woo KS, Chook P, Raitakari OT, et al: Westernization of Chinese adults and increased subclinical atherosclerosis. Arterioscler Thromb Vasc Biol 1999; 19:2487-2493.

33 Lorenz M, Schaefer C, Steinmetz H, et al: Is carotid intima media thickness useful for individual prediction of cardio vascular risk? Ten years results from the Carotid Atherosclerosis Progression Study (CAPS). Eur Heart J 2010;31:2041-2048.

-34 Aguilar-Shea A, Gallardo-Mayo C, GarridoElustondo S, et al: Carotid intima-media thickness as a screening tool in cardiovascular primary prevention. Eur J Clin Invest 2011;41:521-526.

35 Shah PK: Screening asymptomatic subjects for subclinical atherosclerosis. Can we, does it matter, and should we? J Am Coll Cardiol 2010;56:98-105.

- 36 Touboul PJ, Vicaut E, Labreuche J, et al: Correlation between the Framingham risk score and intima media thickness: The Paroi Arterielle et Risque Cardio-vasculaire (PARC) study. Atherosclerosis 2007;192:363-369.

37 Nambi V, Chambless L, Folsom AF, et al: Carotid intima-media thickness and presence or absence of plaque improves prediction of coronary heart disease risk: The ARIC (Atherosclerosis Risk in Communities). Study J Am Coll Cardiol 2010;55:1600-1607.

38 Schargrodsky H, Hernández-Hernández R, Champagne B, et al, CARMELA Study Investigators: CARMELA: assessment of cardiovascular risk in seven Latin American cities. AJM 2008;121:58-65.

- 39 Taylor AJ, Burke AP, O’Malley PG, Farb A, Malcom GT, Smialek J, Virmani R: A comparison of the Framingham risk index, coronary artery calcification, and culprit plaque morphology in sudden cardiac death. Circulation 2000;101:1243-1248.

40 Touboul PJ, Hennerici M: Epaisseur intima media AVC et traitements médicamenteux 'IMT, Drugs and Stroke'. Paris, Datebe, 2002, p 108. 